\title{
La pertinencia de las ciencias sociales, en la investigación y la educación en salud, desde una mirada cualitativa
}

The relevance of social sciences, in education and health research, from the qualitative perspective

\section{Volumen 18, Número 3 \\ Setiembre-Diciembre}

pp. 1-21

Este número se publica el 1 de setiembre de 2018

DOI: https://doi.org/10.15517/aie.v18i3.34167

\author{
Hilda P. Núñez Rivas \\ Ileana Holst Schumacher \\ Natalia Campos Saborío
}

Revista indizada en REDALYC, $\underline{\text { SCIELO }}$

Revista distribuida en las bases de datos:

LATINDEX, DOAJ, REDIB, IRESIE, CLASE, DIALNET, SHERPA/ROMEO, QUALIS-CAPES, MIAR

Revista registrada en los directorios:

ULRICH'S $, \underline{R E D I E}, \underline{\text { RINACE}}, \underline{\text { OEI }}, \underline{\text { MAESTROTECA }}, \underline{\text { PREAL, }}$ 


\title{
La pertinencia de las ciencias sociales, en la investigación y la educación en salud, desde una mirada cualitativa \\ The relevance of social sciences, in education and health research, from the qualitative perspective
}

\author{
Hilda P. Núñez Rivas ${ }^{1}$ \\ Ileana Holst Schumacher ${ }^{2}$ \\ Natalia Campos Saborío 3
}

\begin{abstract}
Resumen: En el marco de la teoría de los intereses constitutivos de saberes de Habermas, las ciencias de la salud no han sido posicionadas, de manera formal en ella para explicar, comprender y transformar sus objetos o fenómenos de estudio a profundidad. Los gremios científicos de las ciencias de la salud han analizado estas ciencias y llevado a cabo los procesos de formación, capacitación y educación continua de su personal con el método científico tradicional del positivismo y han relegado el uso de otros métodos científicos "naturalistascualitativos" aportados por las ciencias sociales. El propósito de este ensayo es delinear cuatro aspectos teóricometodológicos que justifican el uso de métodos mixtos, por lo que se proveen experiencias de investigación con esta metodología. Se concluye que, no es el uso de una técnica particular la que define a una persona investigadora como positivista o interpretativa o sociocrítica, sino que es preciso comprender la existencia de una correlación entre el paradigma y los métodos de investigación cuantitativos y cualitativos y su empleo en una metodología mixta, en aras de estudiar la realidad de forma holista, ya que no es productivo dar preferencia a uno solo de estos estilos de pensamiento y excluir las potencialidades de otros. En consecuencia, lo primordial y necesario es examinar la realidad integralmente, con metodologías pluralistas para potenciar su cambio hacia condiciones de mayor equidad y justicia social.
\end{abstract}

Palabras clave: ciencias sociales, ciencias de la salud, metodología, investigación científica, Habermas.

\begin{abstract}
In the framework of Habermas' theory of knowledge constitutive interests, the health sciences have not been formally positioned in it to explain, understand and transform their objects or phenomena of study in depth. The scientific societies of health sciences have analyzed these sciences and carried out the training and continuing education of their personnel using the traditional positivist scientific method and have relegated the use of other scientific methods "naturalistic-qualitative" contributed by the social sciences. The purpose of this essay is to outline four theoretical-methodological aspects that justify the use of a mixed methodology and provide research experiences with these methods. It is concluded that it is not the use of a particular technique that defines a researcher as positivist or interpretative or sociocritical, but it is necessary to understand the existence of a correlation between the paradigm and the quantitative and qualitative research methods and their use in a mixed methodology, in order to study reality in a holistic way. It is not productive to give preference to only one of these styles of thought and to exclude the potential of other ones. Consequently, the essential and necessary thing is to examine reality in its entirety, with pluralistic methodologies to promote its change towards conditions of greater equity and social justice.
\end{abstract}

Key words: social sciences, human, health, methodology, scientific research, Habermas.

\footnotetext{
1 Instituto Costarricense de Investigación y Enseñanza en Nutrición y Salud (INCIENSA), Costa Rica. Dirección electrónica: hnunez@inciensa.sa.cr

2 Universidad de Costa Rica (UCR), Facultad de Microbiología. Dirección electrónica: ileana.holst@ucr.ac.cr

${ }^{3}$ Universidad Estatal a Distancia (UNED), Costa Rica. Dirección electrónica: nataliacampos07@yahoo.com
}

Ensayo recibido: 31 de octubre, 2017

Enviado a corrección: 13 de marzo, 2018

Aprobado: 21 de mayo, 2018 


\section{Introducción}

El conocer es exclusivo de los seres humanos porque son los únicos seres vivos que tienen coeficiente intelectual, es decir capacidad de raciocinio. El conocimiento es una representación social de la existencia de la realidad, es un producto de la interacción entre el ser humano y la realidad (el objeto o fenómeno de estudio). Existen varios tipos de conocimiento: científico, empírico, tecnológico, técnico, artístico, religioso y mítico (Acevedo Borrego, Linares Barrantes, Cachay Boza, 2014).

En este ensayo se hace énfasis en el interés empírico, producto de la experiencia, y en el interés científico, generado o mediado por la aplicación de algún método científico. Se aclara que no existe solo un método científico, aquel tradicional, experimental e hipotéticodeductivo, que parte de la formulación de hipótesis sobre la realidad estudiada, la cual se somete a contrastación y cuyos pasos se inician con la observación, descripción y finaliza con leyes universales (Carbonelli, Cruz e Irrazábal, 2011). Este método "tradicional" es el que aún se enseña en las escuelas, los colegios e incluso las universidades; es el que las y los científicos positivistas conciben como válido y verdadero. No obstante, existen otros métodos del paradigma cualitativo que aportan datos válidos y confiables (Noreña, Alcaraz-Moreno, Rojas, Rebolledo-Malpica, 2012).

Las ciencias, de acuerdo con los objetos o fenómenos de estudio, emplean diferentes métodos científicos para conocerlos. El uso del método tradicional y/o de otros métodos científicos dependerá en parte del sistema de creencias que posean las personas investigadoras y de la naturaleza del objeto de estudio o fenómeno (Vázquez, Acevedo, Manassero y Acevedo, 2001). De ahí que, los objetos o fenómenos de estudio son construcciones que los individuos investigadores han aportado a las ciencias para explicarlas e interpretarlas. Estas construcciones a su vez justifican la existencia de las ciencias. Por ejemplo, los nutrientes son "objetos de estudio" que la ciencia de la nutrición humana ha construido, mediante teorías explicativas sobre sus estructuras, funciones y relaciones con la bioquímica y la fisiología del ser humano.

Estas construcciones teóricas, en mención, cambian, se transforman y se enriquecen conforme se genera conocimiento científico y se renuevan los intereses y las necesidades de comprender/explicar los nutrientes-objetos de estudio. De manera que, otros factores o aspectos socioculturales y psicosociales podrían influir en la dieta. Por ejemplo, la bomba calorimétrica, invento de Marcelin Pierre Eugène Berthelot del siglo XIX (Químico francés. París, 1827 - 1907) cuya función fue estudiada con el enfoque técnico, en la actualidad se 
usa para explicar también las relaciones entre la microbiota intestinal humana y el metabolismo corporal, por sus implicaciones con la obesidad y la diabetes (Jumpertz et al., 2012). Por lo tanto, se puede afirmar que fue la óptica positivista la que prevaleció en la selección del método científico y dependió del objeto de estudio y del sujeto que investigó. Pudo ser desde la óptica de la ciencia exacta o de la ciencia social. Así también, dos o más investigadores que estudian este mismo objeto de estudio, por sus sistemas de creencias, podrían seleccionar métodos diferentes para dar respuesta al problema de investigación. Es en ese sentido que Habermas (1982) mediante su teoría de los intereses constitutivos de saberes, propone que existen tres diferentes formas de acción ante la realidad a estudiar, cada una responde a un tipo de interés cognitivo: técnico, práctico o emancipador (Grundy, 1991). Con esta teoría publicada desde la década de los sesentas, Habermas rechaza la idea de que los saberes son generados únicamente por procesos intelectuales productos de la capacidad de raciocinio de las personas investigadoras.

Habermas sostiene que las y los sujetos investigadores no son excepciones de actitudes desinteresadas. Al contrario, los intereses constitutivos de saberes anteceden e influyen directamente en los actos cognoscitivos y modelos de pensamientos de las personas investigadoras, los que, a su vez, inciden en las decisiones y elecciones sobre las formas de construcción de la realidad y las actuaciones. Por ello, para Habermas y también para Husserl, el positivismo científico niega u olvida la reflexión del contexto en donde se estudia el objeto o fenómeno (Hoyos Vásquez, 2011). Desde esta perspectiva, la objetividad de las y los científicos está impregnada de cierta subjetividad.

Cabe destacar que cada interés cognitivo se desarrolla y cristaliza no sólo en los procesos de investigación científica, sino también en los contextos donde se desenvuelve el ser humano. En esos contextos está presente también el conocimiento empírico, del cual surgen ideas y problemas de investigación, que serán resueltos de acuerdo con los intereses de las personas investigadoras. Aquellas guiadas por el interés técnico se concentrarán en los problemas de estudio propios de las ciencias analítico-empíricas; aquellas personas orientadas por el interés práctico, se centralizarán en la interacción humana y abordarán problemas de estudio de las disciplinas hermenéutico-históricas y las guiadas por el interés emancipador o emancipatorio, investigarán problemas de las ciencias socio-críticas, que analizan aquellas condiciones socio-históricas estructuradas y estructurantes del objeto o fenómeno en estudio. 
En el marco de la teoría de los intereses constitutivos de saberes de Habermas, las ciencias de la salud no han sido posicionadas en ella, de manera formal como elementos fundamentales de discusión para explicar, comprender y transformar sus objetos o fenómenos de estudio a profundidad. Así también, los gremios científicos de las ciencias de la salud, con énfasis en lo clínico y lo epidemiológico, han analizado a estas ciencias y llevado a cabo los procesos de formación, capacitación y educación continua de su personal, con el método científico tradicional del positivismo, que es más empleado en las ciencias exactas; y han relegado el uso de otros métodos científicos "naturalistas-cualitativos" aportados por las ciencias sociales.

No obstante, a partir del siglo XXI a nivel internacional, los métodos cualitativos y posteriormente los mixtos se han utilizado en las ciencias de la salud y se ha discutido acerca de los fundamentos filosóficos, principalmente epistemológicos, de los paradigmas positivista e interpretativo subyacentes en estos métodos (Creswell, Klassen, Plano Clark y Smith, 2011; Hesse Biber, 2015). El propósito de este ensayo es delinear cuatro aspectos teórico-metodológicos que son de utilidad para justificar el uso de métodos mixtos, no sin antes hacer mención del aporte del paradigma cualitativo y las ciencias sociales, para responder a preguntas de investigación de las ciencias de la salud. Asimismo, las autoras proveen experiencias de investigación con métodos mixtos; ellas conforman un equipo interinstitucional e interdisciplinario enmarcado en el estudio de la obesidad y promoción de la salud integral de niños y adolescentes en Costa Rica.

\section{Proposición}

Este ensayo apunta a cuatro aspectos teórico-metodológicos polémicos que deben ser retomados en una agenda de discusión de las disputas epistemológicas entre lo cuantitativo y lo cualitativo. Se debe valorar su pertinencia, tanto en la investigación científica como en la formación, la capacitación y la educación continua del personal de salud. Lo anterior facilitará el posicionamiento del fenómeno de estudio de las ciencias de la salud, de manera que la investigación social y educativa no continúe "acaparada" por el positivismo científico. La investigación multimétodo en salud es considerada necesaria y pertinente, ya que las personas investigadoras tienen diferentes puntos de vista filosóficos, lo que favorece la capacidad para responder a preguntas complejas.

Los cuatro aspectos seleccionados para este ensayo son: 
1. El paradigma positivista y su influencia en el modelo médico hegemónico, que tiene implicaciones en la investigación científica de la salud humana y la formación, la capacitación y la educación continua del personal de salud

2. El proceso salud-enfermedad como objeto/fenómeno de estudio de las ciencias de la salud, en el marco de los enfoques interpretativos de los intereses de Habermas

3. La triangulación de resultados de investigación en salud mediante los enfoques práctico (interpretativo), sociocrítico y técnico

4. La formación, la capacitación y la educación continua del personal de salud con aportes de las ciencias sociales con énfasis en la teoría de los intereses constitutivos de saberes de Habermas

\section{La influencia del paradigma positivista en el modelo médico hegemónico}

El uso del método empírico-analítico o experimental en la investigación científica de la salud humana obedece a una lucha de poder hegemónico sobre la validez y la legitimización del conocimiento (Adames Mayorga, 2007). Las ciencias de la salud humana, representadas en la medicina, así como la biología, la química y la física, han sido fieles a este paradigma positivista. El apartarse totalmente, o incluso, de manera parcial del paradigma positivista, implica que las ciencias sean cuestionadas por su validez y legitimación científicas.

En la investigación en salud se sobrevalora la necesidad de generalizar los datos y de llegar a leyes universales, mediante el uso de técnicas cuantitativas. La balanza se posiciona más sobre la validez externa de los resultados de una investigación en salud que sobre la validez interna, dada la necesidad de que los resultados sean extrapolados a la población general, pues es a esta a la que se dirigen las políticas, los programas e intervenciones nacionales en pro de la salud pública.

Las técnicas cualitativas no tienen como fin generalizar o extrapolar los datos a la población. Su propósito es profundizar en el fenómeno en estudio, al tomar en cuenta su inserción en una matriz cultural, en donde se generan. Por lo tanto, son particulares y singulares, al considerar que los comportamientos o las conductas de las personas participantes no son homogéneas. La validez de la información se obtiene mediante su triangulación y aporta datos significativos de la realidad estudiada en un contexto particular.

La investigación cualitativa si puede generalizar resultados, mediante los procesos de teorización, pues parten de la indagación teórica existente, hasta llegar a una generalización analítica, es decir, al desarrollo de teoría que ayuda en la comprensión del problema focal en 
otros casos o situaciones similares a la investigación de origen y brinda posibilidades para comparar las diferencias y similitudes entre los casos (Maxwell, 2012; Yin, 2013).

Estas similitudes que en lo cualitativo se llaman "patrones", sirven para "generalizar" lo que los grupos participantes "dan por sentado", porque han sido construidas y compartidas por medio del lenguaje en un contexto cultural de generación en generación y forman parte de la estructura social.

Por ejemplo, en un estudio etnográfico cualitativo realizado por las autoras para comprender a profundidad las creencias sobre obesidad de niños y niñas en edad escolar y las de sus progenitores (Núñez Rivas, Campos Saborío, Alfaro Mora, Holst Schumacher, 2013) se analizaron los siguientes testimonios:

- (...) mi abuelita y yo, eh... mi abuelito, mi mamá y yo estamos como unos embarazados. Las niñas obesas son gordas, cómo es... sudan y comen mucho y los niños obesos también igual, son gordos de peso y comen mucho. La gente ancha es así porque come y come mucho. (Niña de tercer año); (...) si alguien me preguntara si yo soy un niño obeso, yo le diría que sólo estoy un poco pasado del peso que tengo que tener. (Niño de cuarto año); (...) en mi casa todos son gorditos... porque son redonditos, así son ellos, son redondos. En las fotos hay gente que es familia de ellos y bueno mía también (risas) que son así redonditos. (Niña de cuarto año); (...) si alguien me preguntara si yo soy una niña obesa, yo le respondería que sólo un poquito gordita estoy. (Niña de cuarto año). (Núñez Rivas et al., 2013, p. 8).

- (...) oiga, pero ¿sabe una cosa?, todas las gordas son lindas, usted se pone a ver una gorda y es bonita de cara ¿verdad?, aunque el cuerpo sea gorda, pero hay gente que sí le gusta, para todo hay gustos, ¿verdad?... (Padre de una niña con obesidad de cuarto año). (Núñez Rivas, et al., 2013, p. 11)

De las expresiones verbales utilizadas en la cotidianidad de la población de estudio, se logró constatar que:

(...) la población estudiantil no cree que la obesidad sea una enfermedad, pero sí una expresión fenotípica, más que todo a "causa" de que "come y come mucho". De ahí que la población estudiantil le asignó varios sinónimos a la persona con obesidad, tales como: "gorda" o "gordita", "pesada" o "pesadita", "redondita", "ancha" y "gruesa" o "gruesita", entre otros, Núñez (2007). Asimismo, dichas expresiones reflejan que los 
niños y las niñas creen que una persona puede tener obesidad, "pero sólo un poco". También, se puede observar que la obesidad no es una condición deseable, por lo que la mayoría de las niñas, quienes manifestaron que sí estaban "gorditas", agregaron que "sólo un poquito" e incluso un niño de cuarto año, expresó que no lo estaba. Cabe destacar que la población estudiantil con obesidad calificó a sus pares obesos, como "pesados o gordos", pero cuando se autocalificaron, dijeron que "algo pesados" o "un poquito gordos o gordas. (Núñez Rivas et al., 2013, p. 8).

Con los testimonios anteriores se confirma la creencia que tienen los progenitores de que las personas con obesidad poseen un rostro (cara) particular que es agradable, a pesar de que la obesidad es una condición no deseable. (Núñez et al., 2013).

En consecuencia, las ciencias de la salud deben solicitar apoyo y orientación al paradigma interpretativo para comprender que los fenómenos de salud requieren ser entendidos desde los individuos que lo viven en carne propia, pues desde sus cosmovisiones y el uso de lenguaje "común", señalan el rumbo de la salud pública, aspecto que no ofrece el dato cuantitativo.

Por el contrario, el enfoque cualitativo examina con mayor profundidad la realidad a estudiar y los datos se visualizan desde el círculo hermenéutico de lo particular a lo general y viceversa. Por ejemplo, las siguientes preguntas se pueden contestar con el enfoque citado, a saber: ¿Por qué una situación de salud constituye un problema? ¿Por qué no se ha podido prevenir o controlar?

Otras preguntas de tipo metodológico que las personas investigadoras deben considerar para seleccionar un enfoque cuantitativo o cualitativo son las siguientes: ¿Los resultados generalizables son aplicables a grupos "particulares"? ¿Los resultados generalizables serán efectivos para aquellos grupos de riesgo "singulares"? ¿Toda la población comprende el problema de igual manera? ¿Tienen las mismas creencias, costumbres, actitudes y percepciones hacia el problema de salud?

Por otra parte, el enfoque biologista positivista, fragmentado por especialidades, del modelo médico inicia con el diagnóstico físico, clínico y bioquímico, el cual se concibe como resultado dicotómico: es normal o es anormal. Con base en el diagnóstico, la persona dueña de la verdad, desde su racionalidad, define la intervención a seguir la cual consiste en un tratamiento de línea positivista. Este tratamiento parte de la verdad del personal médico o de salud, el cual les otorga a estas personas la autoridad y la legitimación para actuar por 
encima del saber cotidiano. No existen diálogos que contextualicen la realidad social del sujeto (paciente), se mantiene el estado de las cosas y se debilita cualquier intento de modificación de conductas de riesgo para la salud (Bastidas Acevedo, Pérez Becerra, Torres Ospina, Escobar Paucar, Arango Córdoba, Peñaranda Correa, 2009).

Se ha reportado que el personal de salud carece de un bagaje teórico y epistemológico sólido acerca de la comunicación y la educación (Campillo Díaz, Sáez Carreras y Del Cerro Velázquez, 2015), competencias que podrían aprovecharse de las diferentes disciplinas de las ciencias sociales y de las ciencias de la educación, en particular.

Se ha evidenciado la insuficiente comprensión que poseen este personal acerca del saber cotidiano y los contextos, insumos claves para que los procesos preventivos, curativos, comunicativos y educativos no sean de corte informativo-imperativo, conductistas y verticalizados (Bastidas Acevedo et al., 2009). Estos hallazgos evidencian, también, que el paradigma positivista científico ha permeado la formación, la capacitación y la educación continua del personal de salud, mediante el modelo pedagógico tradicional, donde predominan contenidos impositivos y significados unilaterales como dispositivos culturales arbitrales (Bourdieu, 1997).

En este modelo hegemónico-positivista no hay cabida para las realidades internas de los sujetos de estudio respecto a la enfermedad, la salud, el cuerpo, los sentimientos, el dolor, la muerte, entre otras construcciones simbólicas pertenecientes a los diferentes grupos culturales. Estas realidades internas se hegemonizan y no se conciben como procesos sociales sino como conceptos dentro de procesos biológicos, o peor aún, como definiciones dicotómicas de resultados positivos o negativos 0 , que están presentes 0 ausentes. Tampoco se abren espacios para el diálogo interdisciplinario y la equidad en la producción de conocimiento de las ciencias humanas y sociales, pues la epidemiología tradicional compite por la hegemonía en el campo de la salud pública (Magalhães Bosi, 2012).

\section{El proceso salud-enfermedad como objeto/fenómeno de estudio de las ciencias de la salud}

Como punto de partida para la discusión del objeto/fenómeno de las ciencias de la salud, se plantea el proceso salud-enfermedad porque sus determinantes han sido razonadas desde la perspectiva biológica, psicológica y sociocultural y estas no son de génesis espontánea sino socio-histórica. Definir este punto de partida, orienta a buscar un tratamiento integral-holístico complemente al bioestadístico tradicional, pues implica abrirse a 
los aportes teóricos-metodológicos de las ciencias exactas y de las ciencias sociales y en particular de la educación, con enfoques interpretativos y socio-críticos.

Otro rasgo es el carácter socio-histórico del proceso salud enfermedad que integra lo biológico a las particularidades y las singularidades de las personas que están en íntima vinculación con sus grupos sociales de pertenencia y de contextos determinados. La Comisión de Determinantes Sociales de la Salud de la Organización Mundial de la Salud (OMS) considera que estas categorías están relacionadas tanto con las condiciones en las que personas nacen, crecen, viven, trabajan y envejecen, como con aquellos aspectos o factores que son impulsores fundamentales de estas condiciones: la presencia de áreas verdes para la recreación, la disponibilidad y accesibilidad a una alimentación saludable, entre otros que cada día acumulan mayor evidencia científica, en relación con el ingreso, la riqueza y la educación (Braveman y Gottlieb, 2014).

De hecho, estudios han evidenciado que se enferma o se muere de manera diferenciada de acuerdo con la etnia y el país donde se viva (Raza, Snijder, Seidell, Peters y Nicolaou, 2017). Así también, las patologías, las tasas de mortalidad y la esperanza de vida están determinadas por los estratos socioeconómicos de pertenencia dentro de una misma sociedad (Garay y Chiriboga, 2017; Otero, Pechlaner, Liberman y Gürcan, 2015) y algunas patologías están íntimamente relacionadas con las esferas laborales (Nilsen, Andel, Fors, Meinow, Darin Mattsson y Kareholt, 2014).

Por lo tanto, pierde importancia optar por alguna de las dualidades: objetivo-subjetivo, individuo-sociedad, biológico-social. Es decir, la salud física-clínica y bioquímica del individuo, pasa de ser un objeto de estudio objetivo, estandarizado y comparado con parámetros biológicos referenciales, a constituirse en fenómeno de estudio que comprende una serie de procesos sociales dinámicos y de condiciones de vida, donde la evidencia física, clínica y bioquímica del individuo representa una ínfima expresión de su salud integral y bienestar.

Lo anterior, conduce a repensar cómo abordar ese fenómeno social complejo, al considerar conjuntamente las disciplinas de las ciencias médicas (microbiología, farmacia, nutrición, medicina y sus especialidades), duras (física, química), exactas (matemáticas), sociales (antropología, sociología) y de la educación. Esto, a su vez, conduce a la necesidad de responder a las interrogantes de la salud humana, mediante el trabajo en equipo interdisciplinario para mantener una discusión epistemológica del fenómeno de estudio; definir las aristas de los problemas de salud, así como su abordaje, desde diferentes ópticas, 
teorías, métodos y técnicas, hasta alcanzar explicaciones e interpretaciones integrales que generen aportes sustantivos y evidencia científica en pro de la salud humana. Todo ello, debe ser producto de reflexiones serias y rigurosas acerca de los alcances y las limitaciones de las técnicas de investigación utilizadas, sin dejar de lado, su naturaleza de origen o procedencia.

De acuerdo con las experiencias de investigación de las autoras, la madurez del equipo interdisciplinario se reflejará cuando la selección de las teorías, los métodos y las técnicas se realice según las necesidades de explicar o comprender el fenómeno de estudio y sin el interés de mantener la hegemonía de alguna ciencia o disciplina o la subordinación de otras. En el sentido estricto de lo científico, la aplicación de los métodos y las técnicas cuantitativas y cualitativas debieran realizarse con naturalidad, como bien lo aporta Miguel Beltrán Villalba “(...) basta con poner el método cualitativo junto al cuantitativo, dejando que sea el propio objeto de conocimiento el que lo justifique y reclame en función de sus propias necesidades, perfectamente diferenciadas" (1985, p. 34).

Por ejemplo, en una de las investigaciones etnográfica, desarrollada por las autoras para comprender a profundidad las creencias sobre obesidad de estudiantes de la educación general básica, se aplicaron dos instrumentos de frases incompletas con las siguientes preguntas: ¿...las niñas gordas son...? y ¿ ...los niños gordos son...? (Núñez Rivas, 2007)

Las respuestas a dichas preguntas siguieron el análisis que se explicita a continuación: (...) en "el complete" que hicieron por escrito, las 235 niñas y los 225 niños, de segundo a sexto año matriculados en la escuela, caso de estudio, se puede afirmar que se confirmaron los patrones de creencias descritos. El confronto de los datos obtenidos con el instrumento de "frases incompletas" y las entrevistas realizadas, permitió aplicar el círculo hermenéutico (que va de lo general a lo particular y viceversa), contextualizar la información brindada por los niños y las niñas y tener una perspectiva más crítica sobre el fenómeno en estudio. (Núñez Rivas, 2007, p. 153).

(...) las opiniones de los niños y de las niñas son más favorables para los niños obesos que para las niñas obesas. El $40,4 \%$ de los niños y $23,4 \%$ de las niñas opinan favorablemente sobre los niños obesos, mientras que sólo $10,2 \%$ de los niños y $20,4 \%$ de las niñas opinan favorablemente sobre las niñas obesas; existiendo diferencias estadísticamente significativas entre las opiniones de los niños $(40,4 \%$ vrs $10,2 \%, Z=$ 7.260, p < 0,000) (Núñez Rivas, 2007, p. 154). 
Dicho estudio, a pesar de que no privilegió el uso de métodos mixtos, utilizó de forma práctica la combinación de los puntos fuertes de la investigación cuantitativa y cualitativa para abordar problemas de salud "complejos" como la obesidad, lo que aumentó la capacidad de brindar aportes teóricos y prácticos tanto para mejorar la comprensión del desarrollo de la obesidad en la población escolar como la praxis del personal de salud y docente frente a esa problemática (Núñez Rivas, 2007).

El estudio acerca de las creencias de la obesidad de tipo etnográfico es un buen ejemplo para comprender los alcances y las "generalizaciones" que se obtienen a partir del método cualitativo, pues esta investigación permitió develar y construir las creencias acerca de la obesidad de una comunidad educativa. Esta comunidad se constituye en la expresión general de los grupos con características singulares (estudiantes, padres de familia 0 encargados, personal docente y administrativo) que la conforman, dentro un orden social particular. Asimismo, la comunidad educativa está inmersa dentro de un contexto educativo determinado, el cual es el sistema de educación formal de Costa Rica (Núñez Rivas, 2007).

\section{La triangulación de resultados de investigación en salud}

En los puntos anteriores se ha justificado la importancia de considerar los enfoques interpretativo y sociocrítico en el estudio de las ciencias de la salud en su desarrollo histórico y no privilegiar, únicamente, el interés técnico. La comunidad de personas investigadoras ha planteado la teoría de los métodos mixtos, cuyas técnicas de investigación recolecten datos de naturaleza cuantitativa y cualitativa, de manera que se analice el objeto o fenómeno en estudio de forma más completa. A principios del siglo XXI, la investigación de métodos mixtos se ha presentado como una tercera opción de cambio de enfoque (Denzin 2010; Giddings, 2006) y se refiere a cualquier investigación donde coexisten componentes cualitativos y cuantitativos (Onwuegbuzie y Collins 2007; Wisdom, Cavaleri, Onwuegbuzie y Green, 2012), con la salvedad de que en algunos estudios podría predominar uno de esos dos componentes (Creswell, 2013).

Los métodos mixtos representan un cambio en la cultura de la investigación, donde se da la aceptación de la subjetividad de las personas participantes y se valora lo complejo de la investigación científica para contestar las interrogantes sobre la salud "pública". Estas respuestas requieren no solo la explicación del método positivista, sino la comprensión del fenómeno a profundidad en su contexto, para encontrar soluciones pertinentes a los problemas de salud. 
En salud, algunos podrían opinar que se trata de una falsa dicotomía entre un enfoque riguroso basado en el positivismo científico y un enfoque cualitativo basado en el paradigma interpretativo, que consiste en procedimientos complejos para la captura, el análisis de los datos y la generación de las conclusiones. No obstante, este último en la actualidad es quizás de gran utilidad para comprender las raíces y las consecuencias de los problemas de salud "positivizados" (Núñez Rivas et al., 2013). Se hace referencia a los problemas de salud pública como la obesidad, fenómeno complejo que ha sido cuantificado en su magnitud (incidencia y prevalencia) y explicado en tiempo y persona (Núñez Rivas, Monge Rojas, León León y Roselló Araya, 2003).

El considerar que la obesidad es un fenómeno complejo significa tener una visión holística de este problema de salud pública ya que se reconoce que requiere la comprensión de múltiples factores biopsicosociales que tienen orígenes genéticos e históricos. Y si suma a esto, el análisis de las determinantes socioculturales de la obesidad, como la pobreza, el ambiente y la educación, "la ecuación multinivel de la comprensión de la obesidad" se vuelve aún más compleja y exige que su estudio tenga un abordaje holístico y no solamente físicoantropométrico y bioquímico.

Se debe prestar atención a que las metodologías científicas son consideradas como componentes independientes, al diseñar un estudio que desea responder a una pregunta de investigación. No obstante, las autoras de este ensayo consideran que estas metodologías se pueden integrar, siempre y cuando se mantengan sus características únicas y el rigor de cada método, para generar resultados que son también válidos y más abarcadores e integrales que las partes individuales.

Para ilustrar con un ejemplo, se comenta el uso del método mixto en una investigación sobre características psicosociales de estudiantes nicaragüenses en el contexto de la educación intercultural, (Campos-Saborío, Núñez-Rivas, Holst-Schumacher, Alfaro-Mora, Chacón-Ruiz, 2018) la cual se realizó en dos etapas: se utilizaron técnicas de investigación cuantitativas para fines descriptivos en la primera etapa y técnicas cualitativas en la segunda etapa para profundizar en el análisis de los resultados cuantitativos obtenidos durante la primera etapa. Las variables psicosociales y socioculturales de una muestra nacional y probabilística de 2667 niños y adolescentes migrantes y costarricenses del sistema educativo se abordaron desde dos perspectivas: 1) las cuestiones estructurales que emanan de las políticas del Estado hacia los y las migrantes y 2) las respuestas de la población receptora o migrante. Así también, se llevó a cabo el análisis del contenido de los 
documentos relativos a los derechos humanos para la democracia y la paz del Ministerio de Educación Pública de Costa Rica. El diseño mixto arrojó resultados cuantitativos (estadísticos descriptivos y multivariados) y cualitativos conformados por los testimonios de los y las estudiantes y el análisis de documentos relacionados con la educación intercultural y los derechos humanos.

Es importante destacar que la decisión del uso del método mixto conllevó una discusión de su pertinencia con la temática, en el seno del equipo de investigación para la definición de las preguntas del estudio, previa elaboración del estado del arte de la educación intercultural. Una vez definidas las preguntas, continuaron los procesos de reflexión acerca de estas interrogantes y cada investigadora aportó a la discusión, desde su formación profesional (ciencias de la educación, ciencias de la salud y ciencias sociales) y experiencia en investigación.

No obstante, a pesar de que los métodos seleccionados se dividieron en el tiempo del proceso de investigación bietápica, los resultados obtenidos no se encapsularon por método o por su naturaleza cuantitativa o cualitativa, sino que se convirtieron en límites permeables donde dos o más métodos fueron usados conjuntamente para responder las preguntas de investigación de manera más completa y abarcadora. Esto se puede apreciar en los resultados obtenidos a partir de los modelos de regresión logística y de las respuestas a preguntas abiertas y las entrevistas a profundidad acerca del tema autoagresión deliberada en el marco de la educación intercultural de escuelas y colegios de Costa Rica.

Es así como, se utilizaron datos cuantitativos y cualitativos de la siguiente manera: a partir de la regresión logística, el perfil de los y las estudiantes nicaragüenses muestra que es más probable que se lastimen y tengan 3.5 veces más deseos de morir que los y las estudiantes costarricenses ( $\mathrm{OR}=3.578$, IC 95\%, 1.492-8.500) (Campos-Saborío et al., 2018). Este dato se completó con testimonios de estudiantes nicaragüenses que a la letra dicen:

(...) otro estudiante nicaragüense expresó la frustración que sentía: "mm ...mi preocupación es que nadie me escucha, ah nadie le intereso!! ¡Todos dicen que soy una persona rara a mí me da igual...!! Lo que quiero es dejar de existir, ok gracias!! (Campos-Saborío et al., 2018). 
Por lo tanto, el uso de la metodología mixta en dicho estudio permitió brindar un panorama integral y holístico acerca del papel de la educación intercultural en Costa Rica. A manera de ilustración, este estudio develó que:

(...) muchos niños y niñas nicaragüenses en Costa Rica están culturalmente integrados

e integradas en el sistema de educación primaria y secundaria pero socialmente excluidos y excluidas, lo que resulta en baja motivación para el estudio y pocas aspiraciones o expectativas de movilidad social. Los niños y las niñas nicaragüenses se convierten en camaleones tratando de adaptarse a los ambientes que habitan, pero cambiando a la apariencia física más ventajosa, a menudo negando su propio color y disfrazados para evitar el rechazo. (Campos-Saborío et al., 2018).

Por lo anterior, las autoras de este ensayo consideran que la importancia del uso de métodos mixtos radica en utilizar las fortalezas de cada enfoque, de tal manera que ofrezca respuestas integrales a la problemática planteada, desde el dato cuantitativo y cualitativo.

\section{La formación, la capacitación y la educación continua del personal de salud con aportes de las ciencias sociales}

Retomar el enfoque biologista-positivista del modelo médico-hegemónico, que es fragmentado por especialidades, implica considerar en el análisis a la educación para la salud, cuyos procesos de capacitación y educación continua, se plantean desde una racionalidad positivista y orientada por una lógica hipotética-deductiva, técnica y lineal. Esta racionalidad afecta principalmente, la formación de pregrado y postgrado de los profesionales en salud, quienes laborarán en investigación o en docencia en centros educativos, instituciones de educación superior o especializadas en investigación en salud. Se afirma que algunas reformas en salud no ha sido efectivas porque no fueron concebidas para romper ese monopolio positivista, en el sentido de que no promovieron la intervención cultural o educativa (Adinolfi, 2014)..

Estas influencias del modelo médico-hegemónico no permiten el desarrollo de ambientes democráticos y obstaculizan la comunicación asertiva entre el recurso humano educador en salud y la población, los diálogos entre los saberes científico y cotidiano y las actitudes reflexivas sobre los significados acerca del ser humano y su valor por lo que es y su cultura (Vetere, 2006). 
En consecuencia, se asume que el diálogo entre los saberes científico y cotidiano se da en el marco de una propuesta humanista, por lo que la formación, la capacitación y la educación continua del recurso humano en salud debe encauzarse al reconocimiento del otro como sujeto con capacidades y derechos, con voz y voto para decidir acerca de su salud, de acuerdo con sus condiciones biopsicosociales y económicas. Se requiere que en los procesos formativos los profesionales en salud, los educandos y los usuarios (más conocidos como "pacientes") se reconozcan como seres humanos integrales con sensibilidad social y responsables de sus decisiones y acciones en pro de la salud pública. Asimismo, es necesario que se promueva, entre esos actores sociales, la tenencia de conciencia acerca de la desigualdad social (conciencia crítica) (Zaidi, Verstegen, Vyas, Hamed, Dornan y Morahan, 2016).

Las autoras de este ensayo consideran que la educación dialógica en la formación, la capacitación y la educación continua de los recursos humanos en salud, sería un aporte valioso de las ciencias sociales, desde el interés sociocrítico. Freire $(1977,1997,2002)$ y Gadotti (2003) partieron del individuo ya inmerso en la colectividad y en la lucha que obviamente encierra el contexto histórico, un individuo que se ve comprometido a "ser más" en un mundo en que es oprimido por grandes contradicciones. De acuerdo con ambos autores, la educación dialógica no es solamente un derecho de todos, sino el único instrumento para la "salvación" de la tragedia social. No se trata, entonces, de mera instrucción o de entrega de recetas, sino de un proceso cargado de contenidos históricos, humanos, producto de la sensibilidad, la experiencia y la reflexión (Schön, 1983, 1987).

Las disputas epistemológicas entre las ciencias de la salud y las ciencias sociales con enfoques interpretativos y socio-críticos han dejado de tener sentido dado que, tanto en la investigación científica como en la formación, la capacitación y la educación continua, se reconoce que los conocimientos científicos no son datos fríos ni descontextualizados y el cúmulo de evidencia científica es cada día más global que local y más temática que disciplinar. Los productos generados a partir del conocimiento científico son portadores de cultura, esta debe de ser tomada en cuenta y divulgada en todos los niveles, desde las comunidades hasta los grupos gremiales científicos.

Se requiere que ese material científico cultural sea mantenido en su producción y renovado por un recurso humano que se comprometa a promover la movilidad y el dinamismo en las organizaciones de acuerdo con las necesidades en salud pública. Es necesario también que se promueva la proyección de los productos científicos hacia afuera 
de las organizaciones y que se articulen con las comunidades académicas y equipos de investigación de programas y proyectos. En relación con la formación del recurso humano, este debe de educarse, de manera amplia y profunda, con el fin de que los perfiles de salida correspondan a personas académicas/investigadoras críticas sobre las afirmaciones del conocimiento estructurado, desafiar la hegemonía de la ciencia y reconocer las formas disciplinarias de conocimiento que han sido subordinadas o jerárquicamente minimizadas (Rehg y SmithBattle, 2015).

Por lo tanto, se hace necesario integrar en la investigación y en la docencia en salud los diseños mixtos de pluralidad metodológica, el trabajo en equipo interdisciplinario y transdisciplinario para conceptualizar y abordar los problemas con una pedagogía que use un lenguaje común a las ciencias sociales y a las ciencias de la salud.

De esta manera, se podrían abrir espacios académicos y laborales donde el diálogo entre el saber cotidiano de los demandantes de los servicios de salud y el saber "científico" de las y los profesionales oferentes sea fluido y aporte a la comprensión y al abordaje de los problemas de salud de las comunidades.

\section{Síntesis y reflexiones finales}

Ha existido una disputa sobre la conveniencia de utilizar enfoques cuantitativos o enfoques cualitativos en la investigación y en particular, sobre la conveniencia de integrar ambos enfoques en una misma investigación. Estos debates han sido polémicos y en la actualidad se nota un tono más conciliatorio, tendiente a la integración y uso de técnicas de investigación.

La persona investigadora en salud debe explicitar su posición epistemológica, pues esta tiene una fuerte relación con la selección de la metodología que utiliza en su trabajo, al aceptar que una persona investigadora puede integrar técnicas cualitativas y cuantitativas en una investigación y justificarlas en el marco de una clara posición epistemológica con quienes sustentan otras concepciones epistemológicas o que han justificado el uso de metodologías pluralistas.

El empleo de diseños mixtos de pluralidad metodológica en la experiencia investigativa de las autoras de este ensayo ha sido valioso pues permitió:

- Deconstruir preconceptos respecto al objeto/fenómeno de estudio en común y reflexionar sobre los sesgos de abordar el problema según la formación académica base de cada investigadora. 
- Desarrollar la capacidad de diálogo asertivo tanto en la cotidianidad del equipo como en el discurso científico, el cual exige competencias para expresar de manera correcta las conceptualizaciones teóricas relacionadas con el estudio y saber articularlas a lo largo del proceso de investigación.

- Asumir una actitud abierta y no con "prejuicios disciplinarios" a la hora de discutir los métodos de investigación más pertinentes para plantear y abordar el problema de investigación. Esto permitió a las autoras superar los miedos y las preocupaciones respecto al conocimiento y al manejo de métodos y técnicas relativamente "nuevas" para ellas.

- Educarse en fundamentos teóricos y metodológicos diferentes a sus disciplinas, priorizando el buen desarrollo de la investigación y la mayor equidad y la justicia social de población de estudio.

- Renunciar a la convicción de que la forma de construir instrumentos, obtener y analizar los datos de sus disciplinas es suficiente para llevar a cabo la investigación. Esto implicó que cada investigadora "echara mano a la humildad" y madurara la idea de que su aporte era tan valioso como los aportes de sus compañeras de equipo.

- Abdicar al interés de mantener la hegemonía de su ciencia o disciplina, e incluso, de su institución de trabajo o aliarse a la subordinación de otras, tanto en la elaboración de manuscritos científicos como en la presentación de ponencias en actividades científicas.

Como conclusión, se plantea que no es el uso de una técnica particular la que define a un investigador como positivista o interpretativo o sociocrítico. Es preciso comprender la existencia de una correlación entre el paradigma y los métodos de investigación cuantitativos y cualitativos, y su empleo en una investigación de diseño mixto, en aras de estudiar la realidad de forma holista, ya que ningún método tiene patente de exclusividad en la investigación científica. Por lo tanto, no es pertinente dar preferencia a uno solo de estos estilos de pensamiento y excluir las potencialidades de otros. En consecuencia, lo primordial y necesario es examinar la realidad integralmente con metodologías pluralistas para potenciar su cambio hacia condiciones de mayor equidad y justicia social. 


\section{Referencias}

Acevedo Borrego, Adolfo, Linares Barrantes, Carolina y Cachay Boza, Orestes. (2014). Tipos de conocimiento y preferencias para la resolución de problemas. Industrial Data, 13(2). doi: http://dx.doi.org/10.15381/idata.v13i2.6181

Adames Mayorga, Enoch. (2007). Hegemonía y cultura científica. Base para un debate entre ciencias. Revista del Centro de Estudios Latinoamericanos Justo Arosemena, (125), 528. Recuperado de http://studylib.es/doc/8179301/tareas-n\%C2\%B0125

Adinolfi, Paola. (2014). Barriers to reforming healthcare: the Italian case. Health Care Analysis, 22(1). doi: 10.1007/s10728-012-0209-0

Bastidas Acevedo, Miriam, Pérez Becerra, Francy Nelly, Torres Ospina, Julio Nicolás, Escobar Paucar, Gloria, Arango Córdoba, Adriana y Peñaranda Correa, Fernando. (2009). El diálogo de saberes como posición humana frente al otro: referente ontológico y pedagógico en la educación para la salud. Investigación y Educación en Enfermería, 27(1), 104-111. Recuperado de http://www.redalyc.org/articulo.oa?id=105213198011

Beltrán Villalva, Miguel. (1985). Cinco vías de acceso a la realidad social. Revista Española de Investigaciones Sociológicas, (29), 7-42. Recuperado de https://dialnet.unirioja.es/servlet/articulo?codigo $=250532$

Bourdieu, Pierre Félix. (1997). Razones prácticas. Sobre la teoría de la acción. Barcelona, España: Editorial Anagrama. Recuperado de http://epistemh.pbworks.com/f/9.+Bourdieu+Razones+Pr\%C3\%A1cticas.pdf

Braveman, Paula y Gottlieb, Laura. (2014). The Social Determinants of Health: It's Time to Consider the Causes of the Cause. Public Health Reports, 129(1, Suppl 2). doi: $10.1177 / 00333549141291$ S206

Campillo Díaz, Margarita, Sáez Carreras, Juan y Del Cerro Velázquez, Francisco. (2015). El estudio de la práctica y la formación de los profesionales: un reto a las universidades. Revista de Educación a Distancia, (6), 1-23. Recuperado de http://revistas.um.es/red/article/view/245151/185661

Campos-Saborío, Natalia, Núñez-Rivas, Hilda Patricia, Holst-Schumacher, lleana, AlfaroMora, Flory Virginia y Chacón-Ruiz, Betty. (2018). Psychosocial and sociocultural characteristics of Nicaraguan and Costa Rican students in the context of intercultural education in Costa Rica. Intercultural Education. DOI: $\underline{10.1080 / 14675986.2018 .1462957}$

Carbonelli, Marcos, Cruz Esquivel, Juan e Irrazábal, Gabriela. (2011). Introducción al conocimiento científico y a la metodología de la investigación. Buenos Aires, Argentina: Universidad Nacional Arturo Jauretche. Recuperado de https://www.unaj.edu.ar/wpcontent/uploads/2017/02/Introduccion-al-conocimiento-cientifico-y-a-la-metodologia.pdf

Creswell, John W. (2013). Qualitative Inquiry and Research Design: Choosing Among Five Approaches (3a. ed.). Washington DC, EE.UU.: Sage. 
Creswell, John W., Klassen, Ann Carroll, Plano Clark, Vicki L. and Smith, Katherine Clegg. (2011). Best Practices for Mixed Methods Research in the Health Sciences. Washington, DC, EE.UU.: Office of Behavioral and Social Sciences Research (OBSSR), National Institutes of Health (NIH). Recuperado de https://www2.jabsom.hawaii.edu/native/docs/tsudocs/Best Practices for Mixed Metho ds Research Aug2011.pdf

Denzin, Norman K. (2010). Moments, Mixed Methods, and Paradigm Dialogs. Qualitative Inquiry, 16(6). doi: http://journals.sagepub.com/doi/pdf/10.1177/1077800410364608

Freire, Paulo. (1977). Pedagogía del Oprimido. México: Siglo Veintiuno Editores, S.A. de C.V.

Freire, Paulo. (1997). Pedagogía de la autonomía, saberes necesarios para la práctica educativa. México: Siglo Veintiuno Editores, S.A. de C.V.

Freire, Paulo. (2002). Pedagogía de la esperanza, un reencuentro con la Pedagogía del oprimido. Buenos Aires, Argentina: Siglo XXI Editores Argentina.

Gadotti, Modacir. (2003). Pedagogía de la praxis. Sao Paulo, Brasil: Instituto Paulo Freire, Diálogos, L’Ullal Edicions.

Garay, Juan E. and Chiriboga, David E. (2017). A paradigm shift for socioeconomic justice and health: from focusing on inequalities to aiming at sustainable equity. Public Health, 149, 149-158. doi http://dx.doi.org/10.1016/i.puhe.2017.04.015

Giddings, Lynne. (2006). Mixed methods research: Positivism in drag? Journal of Research in Nursing, 11(3), 195-203. Recuperado de http://aut.researchgateway.ac.nz/bitstream/handle/10292/3173/Giddings\%20JNR\%20M anuscript\%20Jan\%205th\%202006.pdf?sequence=11

Grundy, Shirley. (1991). Producto o Praxis del Currículum. Madrid, España: Ediciones Morata.

Habermas, Jürgen. (1982). Conocimiento e Interés. Madrid, España: Ediciones Taurus.

Hesse Biber, Sharlene. (2015). The problems and prospects in the teaching of mixed methods research. International Journal of Social Research Methodology, 18(5). doi http://www.tandfonline.com/doi/full/10.1080/13645579.2015.1062622

Hoyos Vásquez, Guillermo. (2011). Los intereses de la vida cotidiana y las ciencias (Kant, Husserl, Habermas). Bogotá, Colombia: Universidad Nacional de Colombia. Recuperado de http://www.javeriana.edu.co/blogs/guillermo hoyos/files/012-Losintereses-de-la-vida-cotidiana-y-las-ciencias.pdf

Jumpertz, Reiner, Le, Duc Son, Turnbaugh, Peter J., Trinidad, Cathy, Bogardus, Clifton, Gordon, Jeffre I. y Krakoff, Jonathan. (2012). Energy-balance studies reveal associations between gut microbes, caloric load, and nutrient absorption in humans. American Journal Clinical Nutrition, 94(1), 58-65. doi: 10.3945/ajcn.110.010132 
Magalhães Bosi, Maria Lúcia. (2012). Productivity and academic assessment in the Brazilian public health field: challenges for human and social sciences research. Cadernos de Saúde Pública, 28(12). doi: http://dx.doi.org/10.1590/S0102-311X2012001400018

Maxwell, Joseph A. (2012). The Importance of Qualitative Research for Causal Explanation in Education. Qualitative Inquiry, 18(8). doi: https://doi.org/10.1177/1077800412452856

Nilsen, Charlotta, Andel, Ross, Fors, Stefan, Meinow, Bettina, Darin Mattsson, Alexander and Kåreholt, Ingemar. (2014). Associations between work-related stress in late midlife, educational attainment, and serious health problems in old age: a longitudinal study with over 20 years of follow-up. BMC Public Health, 14(878). doi: https://doi.org/10.1186/1471-2458-14-878

Noreña, Ana, Alcaraz-Moreno, Noemi, Rojas, Juan Guillermo, Rebolledo-Malpica, Dinora. (2012). Aplicabilidad de los criterios de rigor y éticos en la investigación cualitativa. Aquichan, 12(3), 263-274. Recuperado de http://www.redalyc.org/pdf/741/Resumenes/Resumen 74124948006 1.pdf

Núñez Rivas, Hilda Patricia. (2007). Las creencias sobre obesidad de estudiantes de la Educación General Básica. Educación: Revista de la Universidad de Costa Rica, 31(1). doi: https://doi.org/10.15517/revedu.v31i1.1259

Núñez Rivas, Hilda Patricia, Campos Saborío, Natalia, Alfaro Mora, Flory Virginia y Holst Schumacher, lleana. (2013). Las creencias sobre obesidad de niños y niñas en edad escolar y las de sus progenitores. Revista Electrónica Actualidades Investigativas en Educación, 13(2). doi: https://doi.org/10.15517/aie.v13i2.11738

Núñez Rivas, Hilda Patricia, Monge Rojas, Rafael, León León, Hannia y Roselló Araya, Marlen. (2003). Prevalence of overweight and obesity among Costa Rican elementary school children. Revista Panamericana de Salud Pública, 13(1). Recuperado de https://scielosp.org/article/rpsp/2003.v13n1/24-32/en/

Onwuegbuzie, Anthony J., and Collins, Kathleen M.T. (2007). A Typology of Mixed Methods Sampling Designs in Social Science Research. The Qualitative Report, 12(2), 281-316. Recuperado de http://nsuworks.nova.edu/tar/vol12/iss2/9

Otero, Gerardo, Pechlaner, Gabriela, Liberman, Giselle y Gürcan, Efe Can. (2015). The neoliberal diet and inequality in the United States. Social Science \& Medicine., (142). doi: 10.1016/j.socscimed.2015.08.005

Raza, Qaisar, Snijder, Marieke B., Seidell, Jacob C., Peters, Ron J.G. y Nicolaou, Mary. (2017). Comparison of cardiovascular risk factors and dietary intakes among Javanese Surinamese and South-Asian Surinamese in the Netherlands. The HELIUS study. $\underline{B M C}$ Research Notes, 10(23). doi: https://doi.org/10.1186/s13104-016-2352-4

Rehg, Ellen y SmithBattle, Lee. (2015). On to the 'rough ground': introducing doctoral students to philosophical perspectives on knowledge. Nursing Philosophy, 16(2). doi: 10.1111/nup.12077 
Schön, Donald A. (1983). The reflective practitioner: How professionals think in action. New York, EE.UU.: Basic Books.

Schön, Donald A. (1987). Educating the reflective practitioner: Toward a new design for teaching and learning in the professions. San Francisco, EE.UU.: Jossey Bass.

Vázquez, Ángel, Acevedo, José Antonio, Manassero, María Antonia y Acevedo, Pilar. (2001). Cuatro paradigmas básicos sobre la naturaleza de la ciencia. Argumentos de razón técnica: Revista española de ciencia, tecnología y sociedad, y filosofía de la tecnología, (4), 135-176. Recuperado de http://institucional.us.es/revistas/argumentos/4/art 5.pdf

Vetere, Pablo E. (2006). El Modelo Médico Hegemónico y su reproducción en el Perfil de Médico que promueve la Fac. Cs. Médicas de la U.N.L.P (Tesis presentada para la obtención de grado de Licenciado en Sociología. Universidad Nacional de La Plata. Facultad de Humanidades y Ciencias de la Educación). Recuperada de http://www.memoria.fahce.unlp.edu.ar/tesis/te.562/te.562.pdf

Wisdom, Jennifer P., Cavaleri, Mary A., Onwuegbuzie, Antthony J. y Green, Carla A. (2012). Methodological Reporting in Qualitative, Quantitative, and Mixed Methods Health Services Research Articles. Health Serv Res, 47(2). doi:10.1111/j.14756773.2011.01344.x

Yin, Robert K. (2013). Case study research: Design and methods (5a. ed.). Washington DC, EE.UU.: Sage.

Zaidi, Zareen, Verstegen, Daniëlle, Vyas, Rashmi, Hamed, Omayma, Dornan Tim y Morahan, Page. (2016). Cultural hegemony? Educators' perspectives on facilitating cross-cultural dialogue. Medical Education Online, 21. doi: 10.3402/meo.v21.33145 